\title{
A Comprehensive Dataset from a Smart Grid Testbed for Machine Learning based CPS Security Research
}

\author{
Chuadhry Mujeeb Ahmed ${ }^{1,2}$ and Nandha Kumar Kandasamy ${ }^{1}$ \\ ${ }^{1}$ iTrust, Singapore University of Technology and Design, Singapore, Singapore, \\ ${ }^{2}$ Computer and Information Sciences Department, University of Strathclyde, Glasgow \\ Corresponding Authors: Chuadhry Mujeeb Ahmed (chuadhry@alumni.sutd.edu.sg) \\ and \\ Nandha Kumar Kandasamy (nandha001@e.ntu.edu.sg)
}

\begin{abstract}
Data-sets play a crucial role in advancing the research. However, getting access to real-world data becomes difficult when it comes to critical infrastructures and more so if that data is being acquired for security research. In this work, a comprehensive dataset from a real-world smart electric grid testbed is collected and shared with the research community. A few of the unique features of the dataset and testbed are highlighted.
\end{abstract}

\section{Introduction}

Recent progress in technology is resulting in the digitization of the physical world and things around us. It is expected that communication and computing capabilities will soon be part of all the physical objects [14]. The integration of cyber technologies (computing and communication) with the physical world gives rise to complex systems referred to as Cyber Physical Systems (CPS). CPS has changed the methods that humans used to interact with the physical world. Some examples of CPS are manufacturing, transportation, smart grid, water treatment, medical devices and the Industrial Control Systems (ICS) [17].

CPS is a broad term; in the following, we highlight a major sector applicable to our daily life, that is, the electrical power system as shown in Figure 1. It shows the highlevel architecture of an electrical power system. This is composed of electricity generation (power plants), transmission (electric grid system) and end-users (smart home). As one can imagine this power system is composed of a multitude of devices and physical processes. Power generation and transmission depend on the demand from the utilities and the users. To meet the requirements of the energy demand the critical infrastructure is utilized to ensure a continuous supply of power. Each of the processes in the critical infrastructure is a complex engineering system and needs a sophisticated control to achieve its desired objectives. For example, at the generation stage, we have generators, Intelligent Electronic Devices (IEDs) also incorporating electric relays, all these devices are autonomously controlled by the Programmable Logic Controllers (PLC). This means that we have a lot of sensors monitoring the physical process, actuators/generators and the physical infrastructure that communicate the current physical states with each other and with the PLC. Such communication among smart devices, on one hand, provides flexibility in controlling the complex CPS but the vulnerabilities 
in the same technologies give rise to cyber attacks. In the following, a famous recent attack on a power grid is discussed.

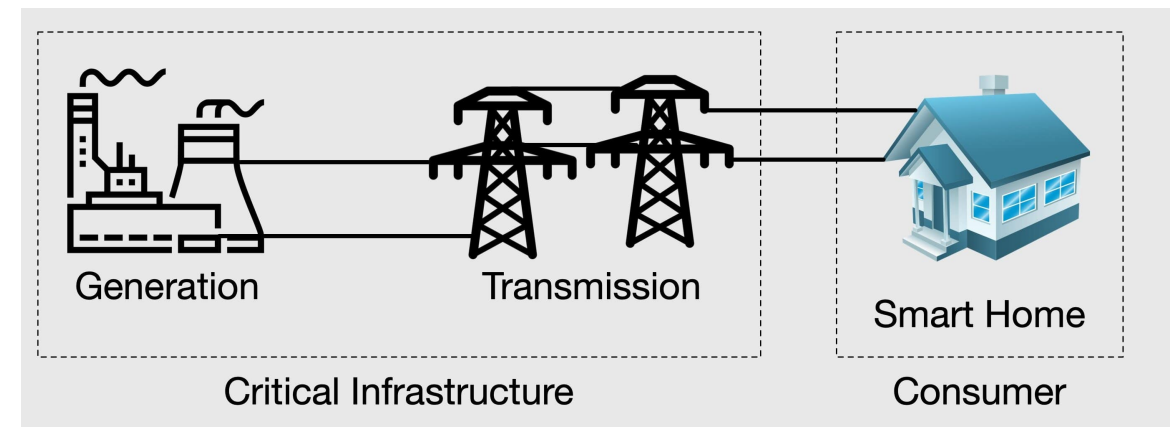

Fig. 1: A generic electrical power system as an example of CPS.

Ukrainian Electric Power Grid Attacks (2015,2016): In December 2015 cyberattacks on Ukrainian electric power grid cut off the power supply to customers at the peak of the winter season. The attackers remotely controlled the SCADA distribution system and forced operators to switch to the manual mode which resulted in much longer recovery times[7]. This attack was over but for another attack to come in the next year around the same time. In 2016 again Ukrainian electric power grid met another cyber attack through the use of Crashoverride malware [18], This attack switched circuit breakers in an unusual open-close pattern in a fast manner, which resulted in cutting off the power supply to the customers. These attacks call for a pro-active approach towards the security of Critical Infrastructure (CI) like power grid.

Successful attacks on CI have led to a surge in the development of defense mechanisms to prevent, contain, and react to cyber attacks. One such defense mechanism is the anomaly detector that aims at raising an alert when the controlled process in a CI moves from its normal to an unexpected, i.e. anomalous, state. Approaches used in the design of such detectors fall into two broad categories: design-centric [1] and data-centric [5].

The use of machine learning to create anomaly detectors becomes attractive with the increasing availability of data and advanced computational resources. However, the data-based techniques rely on a rich dataset representing a real-world scenario. Such datasets are not easily accessible to academia. Our goal is to create a unique set of data that is 1) accessible and 2) represents real-world settings. In this article based on our experiments in a smart electric grid testbed, we have collected data for different states of the physical process under normal and attack conditions.

\subsection{Motivation}

EPIC: Electrical Power and Intelligent Control testbed are used in this study [2] that is a part of other testbeds at iTrust labs [10]. The most notable CPS datasets contributed by iTrust comes from water treatment (SWaT) [12] and water distribution (WADI) [4] 


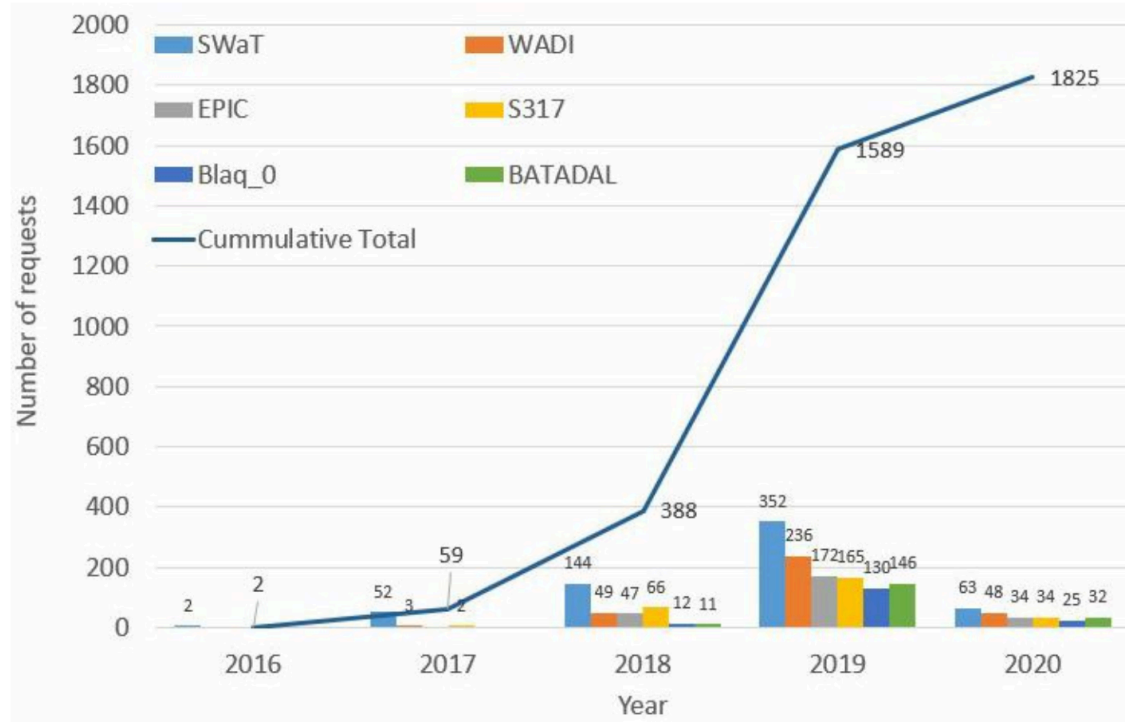

Fig. 2: Overview of dataset requests by year.

testbed. The focus of this work is on the electric grid therefore, we have collected the data for EPIC testbed. To summarize the efforts carried out at iTrust labs on three testbeds Figure 2 shows the requests for the dataset collected at these testbeds for each year. From Figure 2 it can be observed that out of the three testbeds data for EPIC is being requested the least. We hypothesize that could be because there is not a comprehensive dataset for EPIC, unlike other testbeds that explain the data scenarios clearly.

There are some other efforts for data collection beyond iTrust but those still have few limitations. An interesting effort in electric grid testbed is simulation-based Softgrid testbed [9] but there is no data generation and sharing. For an ICS testbed in CPS [8] authors highlighted that their prototype lacked the collection and distribution of data as it involves a manual process requiring time and resources. [6] presented simulated IEC61850 traffic and no information regarding the real process and dynamics.

Previous research studies have tried to collect data from CPS settings but lack some desired features. We highlight a few of those,

- Simulated Data: Most of the datasets available are generated using simulated models [6]. Lack of real-world scenarios prompted us to do this work.

- Only Network Traffic Data: Previous data collection efforts only focused on the network traffic and as mentioned those were too simulated most of the time. One of the recent studies collected the network traffic from a realistic electric traction substation [13]. There is a lack of process data available from an electric grid based on realistic devices.

There have been efforts on building CPS testbeds for security research but very few were able to represent real-world scenarios and collect data to share with the academia and industry. We have focused on the process data from the sensors and actuators and 


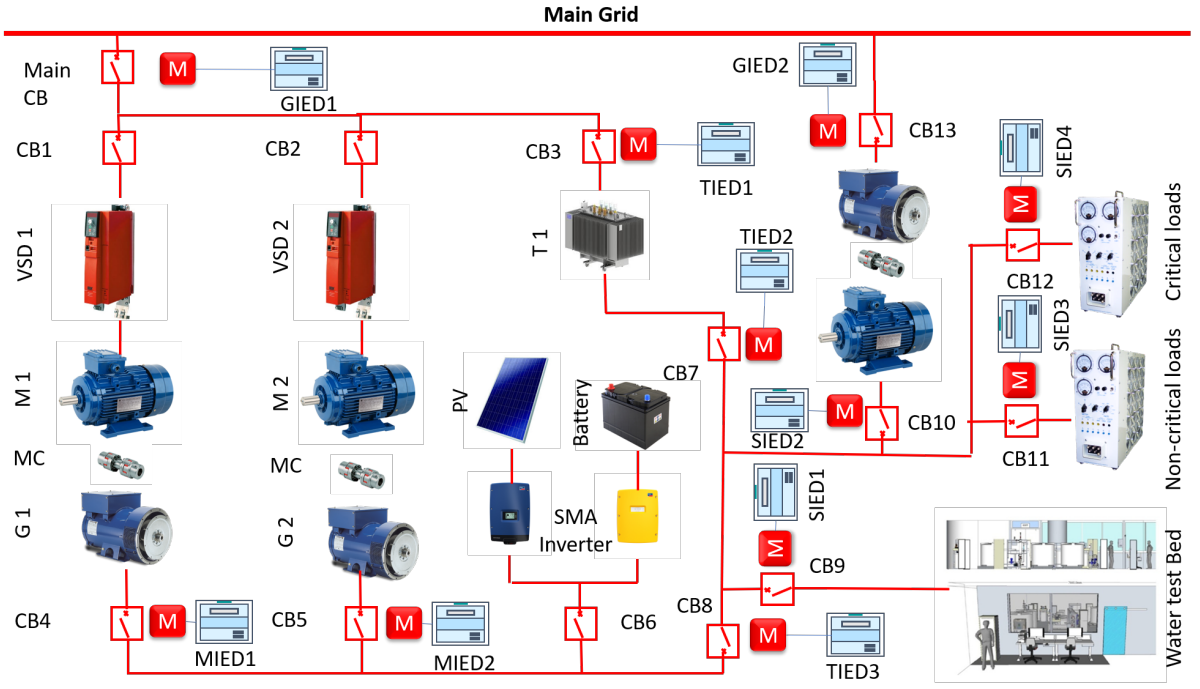

Fig. 3: Electrical layout of the testbed, electrical power lines are shown in red color lines.

tried to run the normal process for a range of different configurations. The idea is if we have enough real-world scenarios for the normal data, it is not hard to generate a malformed data/signal as other researchers have demonstrated in the past, e.g., using the mutation [16].

\section{EPIC Testbed Introduction}

EPIC mainly consists of four zones as described in the following. All the four zones are equipped with IEDs to collect Current, Voltage, Power and Frequency for the three phases buses.

- Generation: Generation stage is driven by electric motors connected to the main power supply.

- MicroGrid: Photo-voltaic cells, inverters and batteries compose this stage to supplement the generation of power.

- Transmission: This stage is composed of buses to transport power to the smart home unit.

- Smart Home: A programmable load bank containing RLC loads represents a home load environment. Besides, there are two water testbeds also connected to EPIC as the load.

\subsection{Electrical Layout}

The testbed consists of the following components as shown in Fig. 3. 1) Two conventional generators (10KVA each) run by $15 \mathrm{~kW}$ VSD driven motors to represent the 
conventional combination of prime-mover and generator. 2) A $34 \mathrm{~kW}$ PV system is available along with an $18 \mathrm{~kW}$ battery system to represent power generation from the intermittent RES. 3) A 105kVA 3 phase voltage regulator for representing power supply from a transmission system. 4) Two load banks capable of emulating 45kVA load to represent critical and non-critical loads. It can supply power to the other two water testbeds. 5) A 10kW motor-generator load to represent spinning load. 6) Industrial standard Molded Case Circuit Breakers are used for short-circuit protection and switching functions.

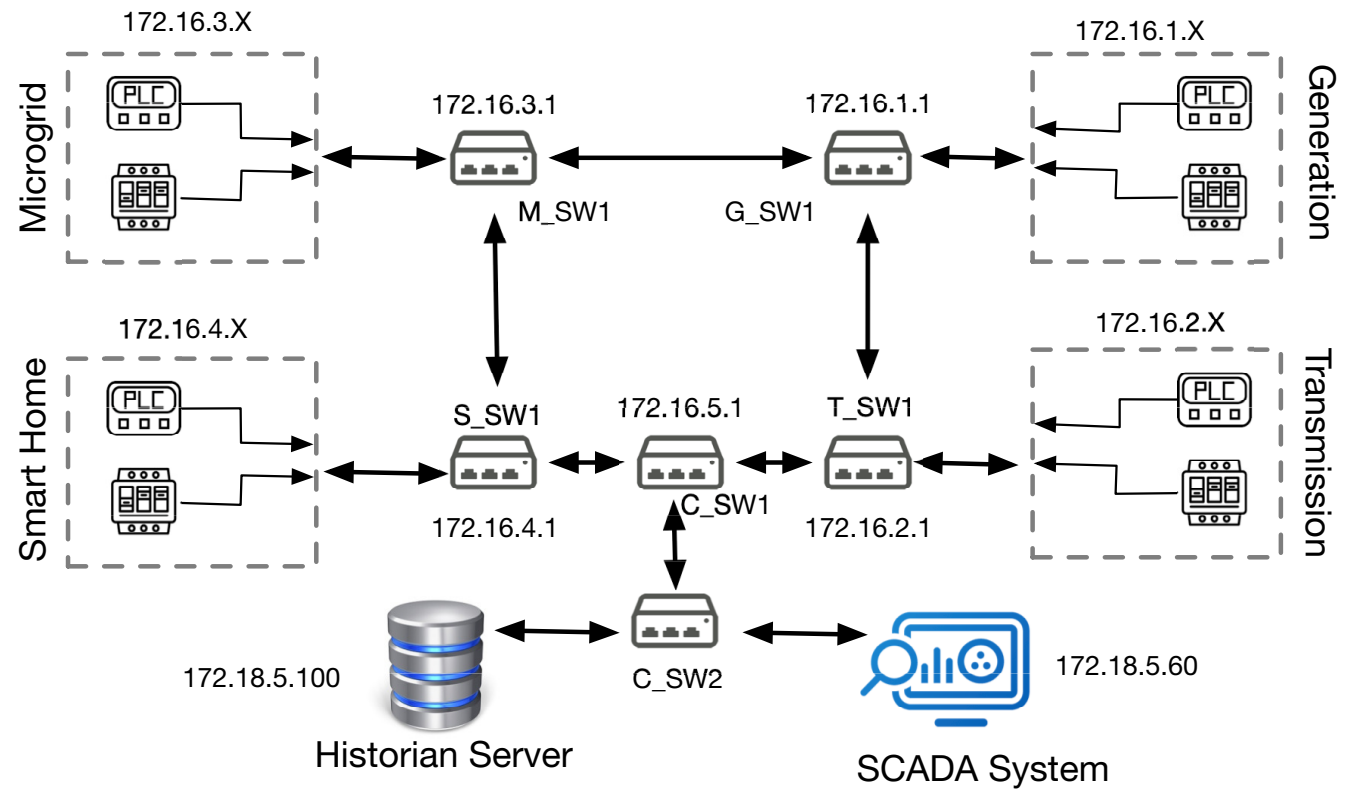

Fig. 4: A simplified network diagram for EPIC is shown. This is to help the associated network traces. IP addresses in the network traces correspond to respected devices shown in the figure. Each dotted box represents a subnet with the respective IP addresses. A X in the IP address means that a device in that subnet would have the similar subnet mask and then unique $\mathrm{X}$ as its own IP. Typical devices are PLCs and Intelligent Electronic Devices.

\subsection{Communication Network}

Figure 4 shows the communication network architecture in EPIC testbed. Figure 4 shows four major control zones, i.e., 1) Power Generation, 2) Transmission, 3) Microgrid, and 4) Smart Home. All of these four zones have IEDs and other devices to be controlled by dedicated PLCs. For example smart home also contains smart meters that can communicate with the PLCs and then can route the data through a central switch to 


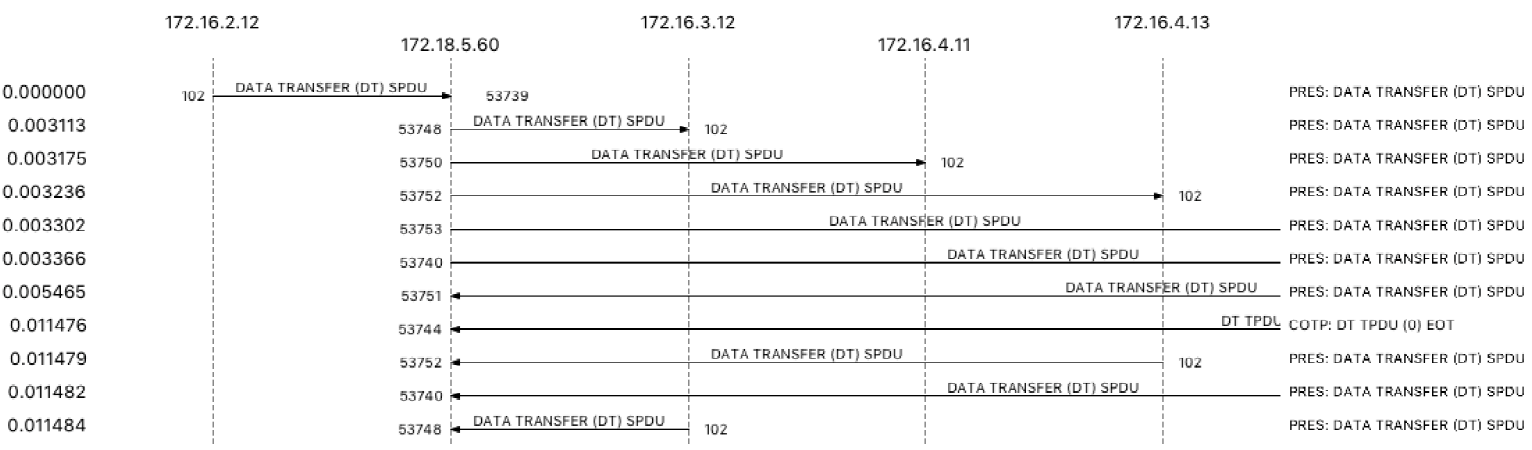

Fig. 5: A sample network traffic flow between different devices.

the historian server and a SCADA workstation. Table 2 in Appendix A shows a map of IP addresses of the specific devices in the testbed. Network traffic is also collected at the SCADA station in a pcap format. Using Table 2 it is possible to make sense of the packet capture of the network traffic. For details on the communication protocols an interested reader is referred to the EPIC testbed papers [15,2]. Figure 5 shows the sequence of communication between the different devices in the EPIC network. In the sequence diagram it can be seen that the SCADA workstation (IP: 172.18.5.60) holds a central position and send data transfer requests to the rest of the PLCs.

\begin{tabular}{|c|c|c|}
\hline Data Header & Explanation & Devices \\
\hline Measurement.Apparent & IED measures the apparent power & GIED, MIED, SIED, TIED \\
\hline Measurement.Frequency & IED measures the signal frequency & GIED, MIED, SIED, TIED \\
\hline Measurement.Line_Current & IED measures the line current & GIED, MIED, SIED, TIED \\
\hline Measurement.V1 & IED measures the voltage difference at L1 & GIED, MIED, SIED, TIED \\
\hline Measurement.Real & IED measures the Real power & GIED, MIED, SIED, TIED \\
\hline Measurement.Reactive & IED measures the Reactive power & GIED, MIED, SIED, TIED \\
\hline Measurement.Power_Factor & IED measures the power factor & GIED, MIED, SIED, TIED \\
\hline Q.mode_close & mode of an electric breaker (T/F) & All Stages \\
\hline VSD.property & properties can be & Generation stage to show the VSD status \\
& .Current,.Speed, .Ready, .Fault & \\
\hline
\end{tabular}

Table 1: Header Explanation in the Data set. IED:= Intelligent Electronic Device, GIED:=Generation stage IED, MIED:= Microgrid stage IED, SIED:= Smart home IED, TIED:= Transmission stage IED.

\section{Data Collection and Process Scenarios}

The data is collected at the different configuration and operational settings of the EPIC testbed. Most of the physical process is driven by the demand from the smart home unit, constituted of different load types. These loads are used to simulate a real-world load 


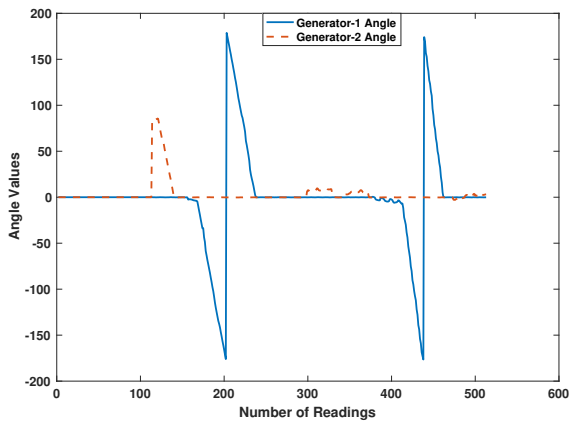

(a) Generator Synchronization without load.

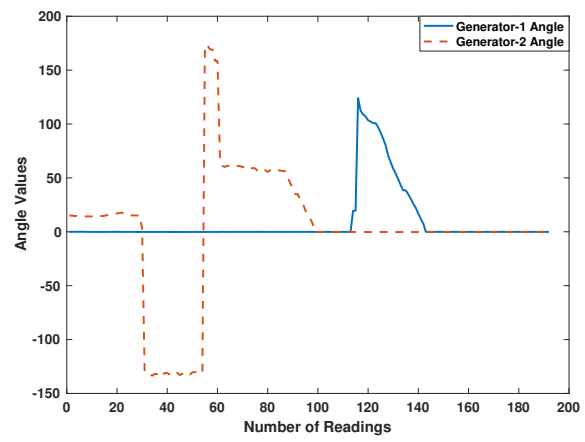

(b) Generator Synchronization with 10KW resistive load.

Fig. 6: Two different smart home load scenarios.

requirement. Moreover, two other water plants are also connected to EPIC as the loads. The collected data can be collected from iTrust labs [10]. Network traffics is collected in pcap format and the process data is provided a Comma Separated Values (CSV) files. To make sense of the process data let us explain what each column in the data files means. Table 1 shows a list of data elements found in the dataset. The first column shows the quantity being measured and the last column shows the devices that are measuring that quantity. For example, the second row has Measurement.Apparent element meaning that the IED is measuring apparent power in different zones of the testbed. A column with header GIED.Measurement.Apparent means the apparent power is measured by an IED at the generation stage. In the following, we highlight the data collection scenarios and settings.

Scenario 1: Synchronization Process without Load This scenario shows the process of synchronization for two generators without any load. Angle difference between two generators G1 and G2 changes in a cyclic manner from -180 to 0 to 180 degree until the synchronization is completed. Figure 6a shows this process twice.

Scenario 2: Synchronization Process with Load To depict the synchronization for the two generators with a $10 \mathrm{~kW}$ resistive load at the smart home stage. Angle difference between two generators G1 and G2 vary from -180 to 0 degrees following a similar cyclic process as in Scenario 1. The additional load had little to no effect on the process. Figure $6 \mathrm{~b}$ shows the process.

Attack on Synchronization Process Synchronization of two generators, a new incoming generator i.e., the generator that needs to be connected in parallel to rest of generators in the grid needs to ensure the following three parameters,

- The frequency of the generator must be same as the frequency of the line/grid

- The magnitude of the generator's voltage must be same as the magnitude of line/grid voltage

- The phase angle of the generator's voltage must be same as the phase angle of the line/grid voltage (we will assume that the phase sequence is same) 

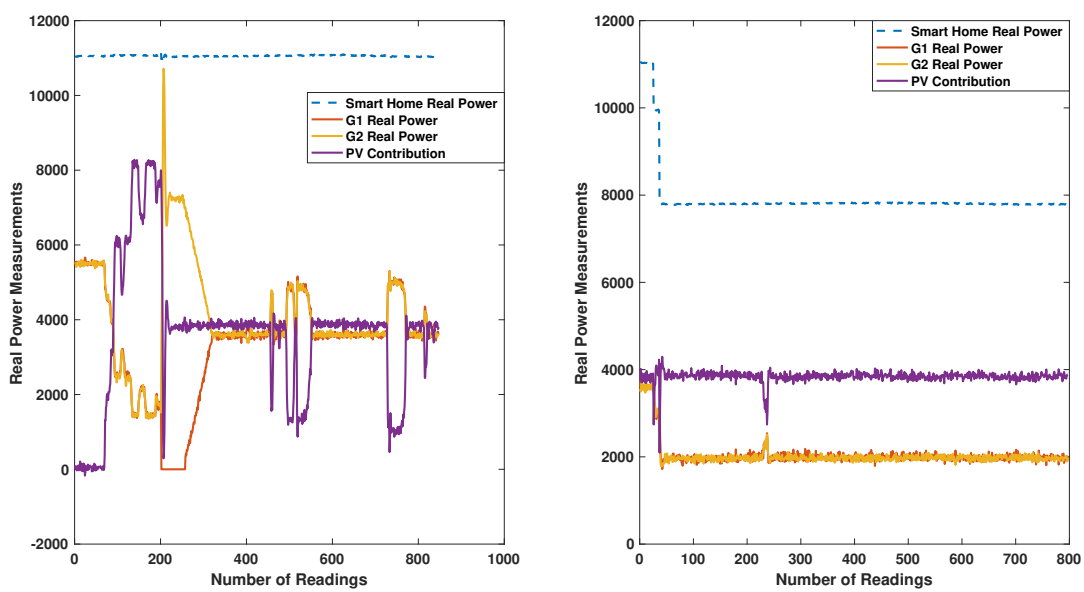

Fig. 7: Scenario-4 with 10KW load and Scenario-5 with $7 \mathrm{KW}$ resistive load.

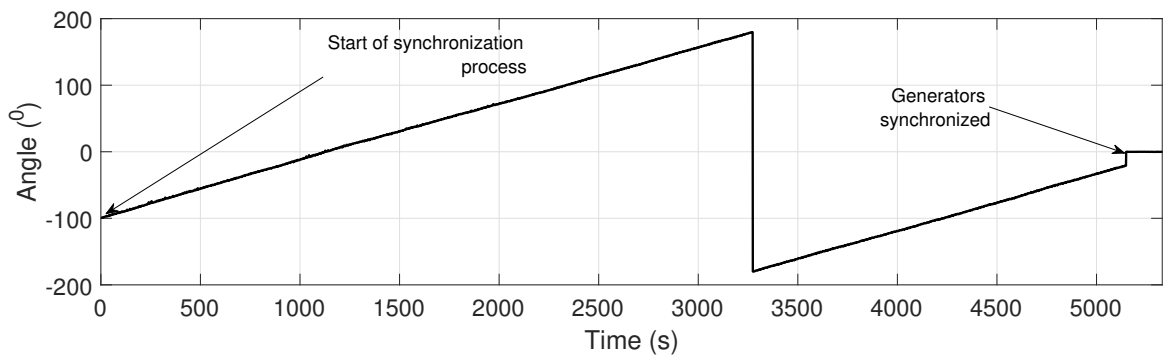

Fig. 8: Experimental results of synchronisation process of two generators after the launch of attack. Normal operation: Observe that $\phi_{d}$ changes from $-180^{\circ}$ to $180^{\circ}$ at significantly slower pace, the breaker is closed when the generators are synchronized which took more than 1.5 hours.

The first two parameters do not depend on the state of the line/grid. However, the third parameters i.e., phase angle depends on the state of the phase angle of the line/grid. The parallel connection is enabled by circuit breaker which closes once the phase angle difference is approximately equal to zero (usually around $10^{\circ}$ in practical cases). We launched the attack on the synchronisation process to delay it from the order of seconds to several minutes. The readers can refer [11] for a detailed analysis on the attack and plausible demand.

Scenario 3: Steady State Behavior Here both the generators are running and synchronized. The resistive load is set to $10 \mathrm{~kW}$ at the smart home stage. This scenario depicts a steady state behavior of the process. Any power plant, substation or section of power system will be in this stage for most of the time.

Scenario 4: PV system with Generators This scenario depicts a situation where the user demand is being fulfilled using the two generators G1 and G2 and also using PV 
system With 10kW resistive load. The scenario depicts the operation of modern power system sections with renewable energy penetration. Figure 7 shows the process behavior for the scenario 4 and 5 respectively.

Scenario 5: PV system with Generators This scenario depicts a situation where the user demand is being fulfilled using the two generators G1 and G2 and also using PV system With a reduced load of $7 \mathrm{~kW}$ resistive load. With decreasing load the chances of creating power supply interruption attacks are relatively high, such scenarios are presented in [3].

Scenario 6: Three Generators Running In this case all the available three generators G1, G2 and G3 are running with a load of $14 \mathrm{~kW}$ resistive load at the smart home stage. This scenario depicts a system where there are motor loads such as buildings with Heat Ventilation and Air-conditioning (HVAC) systems.

Malicious Power Generation Attack- An use case In this case, we manipulated the power generated from one generator to overload it in comparison to the other generator, so that the maintenance schedule can be offset as the overloaded generator needs more frequent maintenance due to additional wear and tear. This eventually leads to accumulated damage in the long run, as the overloaded generator was not serviced at the appropriate times due to malicious operation. During normal operation, to supply power to the critical loads, generators G1 and G2 will share the power equally. The SPLC has the control code that issues a subsequent command to the VSDs to run at a specific speed (1500RPM in this case), for enabling equal power-sharing among the two generators. The apparent power is equally shared between the generators. The time-domain representation of power-sharing before the attack was launched is shown in Figure 9 and marked as normal.

After the attack was launched on generator G1, i.e, the speed of the prime mover of G2 reduced by 0.2 RPM when generator G1 is supplying more power and hence disabling the power-sharing process. This attack scenario is marked in Figure 9 where it can be observed that whenever G2 is supplying more power than G1, G1 takes over until equal power is shared among the two. However, when G1 is supplying more power, G2 fails to take over even after synchronization. This resulted in G1 supplying more power under scenarios where G2 is synchronized as the second generator. The above condition will eventually result in tripping of G1 due to prolonged overload condition.

Scenario 7: Cascading Effects Two generators G1 and G2 running and the load is a real world application. In this case the power is being supplied to the Secure Water Treatment (SWaT) testbed. This scenario can be interesting to see the cascading effects of attack on one CPS system to another. For example, a power supply interruption attack [3] can affect affect the treatment process or the distribution process of water supply system.

Scenario 8: Cascading Effects Two generators G1 and G2 running and the load is two CPS testbeds. In this case the power is being supplied to the Secure Water Treatment 


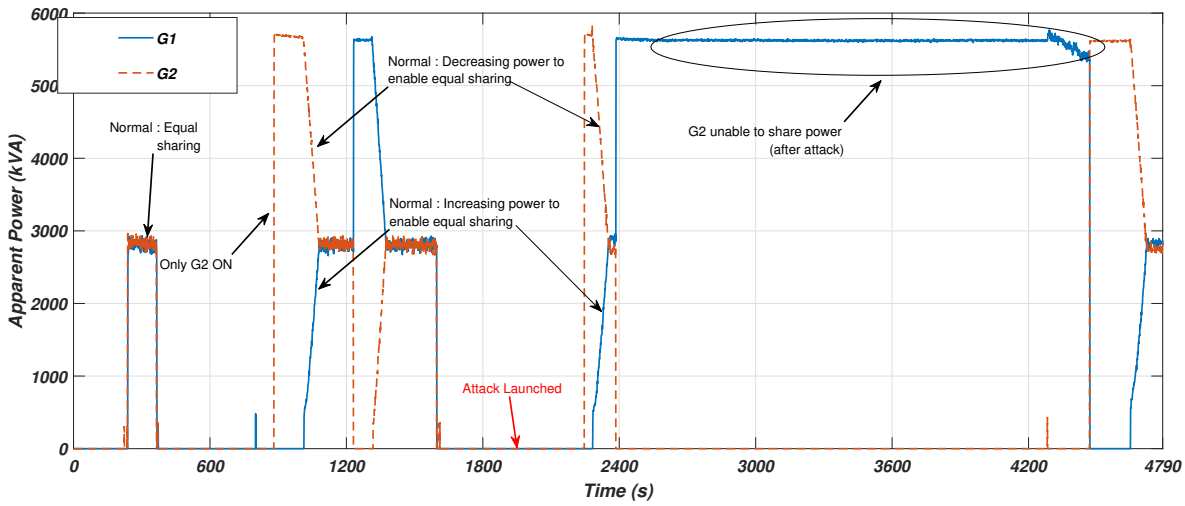

Fig. 9: Apparent power during load sharing between generators G1 and G2. When G2 is unable to share the power, the vulnerability of G1 to trip under overload, i.e., during peak load conditions, increases dramatically. The time-domain representation of power-sharing before the attack was launched is shown and marked as normal. Observe that before attack launch at around 1900 seconds, G1 and G2 shared power irrespective of the condition, whereas after the attack launch, G2 was unable to share the power, i.e., between 2400 and 4300 seconds.

(SWaT) and water distribution (WADI) testbed. This scenario can be interesting to see the cascading effects of attack on one CPS system to another.

\section{Discussion}

It is not possible to collect data for a large number of scenarios and exhaust all the possibilities. We have done it for a range of representative scenarios and then we can use tools like mutation to generate more scenarios for both the normal and abnormal scenarios [16]. However, we tried to present a realistic data from both the process as well as network traffic perspective.

\subsection{Normal Data for Real-World Process}

We have presented the collected data for a range of normal process states from a real world smart electric grid network. It has a very high importance since the quantities measured are across the real process and devices giving an idea of what to expect in real world and come up with the realistic anomaly detection techniques.

\subsection{Lack of Attack Scenarios on the Process}

In this work we have presented the attack scenarios that could be executed without damaging the plant. The attacks those cause physical damage could not be executed on the plant as those pose dangers to the infrastructure and people around it. 


\section{Potential Use Cases and Future Work}

Potential use cases extend from pure network attacks to attacks targeting a particular physical process. For instance, the attacker can explore the network traffic during the circuit breaker closing operation; which can be identified from the process data. Using the data the attacks with physical goal similar to [18] can be re-created. Following which appropriate defense mechanisms could be designed. Similarly, the data on the network traffic and the process status could be coupled for spoofing attacks that can hide the above attack from the operators.

From the defense front, the authors are working on a defense based on negative selection algorithm using dataset for defense against malicious power generation attacks. The dataset is also useful for developing and testing design-centric defense such as invariants based methods and authors are currently working on one such system.

\section{References}

1. Adepu, S., Mathur, A.: Distributed attack detection in a water treatment plant: Method and case study. IEEE Transactions on Dependable and Secure Computing pp. 1-8 (2018)

2. Adepu, S., Kandasamy, N.K., Mathur, A.: Epic: An electric power testbed for research and training in cyber physical systems security. In: Katsikas, S.K., Cuppens, F., Cuppens, N., Lambrinoudakis, C., Antón, A., Gritzalis, S., Mylopoulos, J., Kalloniatis, C. (eds.) Computer Security. pp. 37-52. Springer International Publishing, Cham (2019)

3. Adepu, S., Kandasamy, N.K., Zhou, J., Mathur, A.: Attacks on smart grid: power supply interruption and malicious power generation. International Journal of Information Security pp. 1-23 (2019)

4. Ahmed, C.M., Palleti, V.R., Mathur, A.P.: WADI: A water distribution testbed for research in the design of secure cyber physical systems. In: CPS Week. pp. 25-28. CySWATER '17, ACM (2017)

5. Ahmed, C.M., Zhou, J., Mathur, A.P.: Noise matters: Using sensor and process noise fingerprint to detect stealthy cyber attacks and authenticate sensors in CPS. In: Proceedings of the 34th Annual Computer Security Applications Conference, ACSAC 2018, San Juan, PR, USA, December 03-07, 2018. pp. 566-581 (2018)

6. Biswas, P.P., Tan, H.C., Zhu, Q., Li, Y., Mashima, D., Chen, B.: A synthesized dataset for cybersecurity study of iec 61850 based substation. In: 2019 IEEE International Conference on Communications, Control, and Computing Technologies for Smart Grids (SmartGridComm). pp. 1-7 (2019)

7. Case, D.U.: Analysis of the cyber attack on the ukrainian power grid. Report (2016)

8. Green, B., Lee, A., Antrobus, R., Roedig, U., Hutchison, D., Rashid, A.: Pains, gains and plcs: Ten lessons from building an industrial control systems testbed for security research. In: 10th USENIX Workshop on Cyber Security Experimentation and Test (CSET 17). USENIX Association, Vancouver, BC (Aug 2017), https://www.usenix.org/conference/cset17/workshop-program/presentation/green

9. Gunathilaka, P., Mashima, D., Chen, B.: Softgrid: A software-based smart grid testbed for evaluating substation cybersecurity solutions. In: Proceedings of the 2nd ACM Workshop on Cyber-Physical Systems Security and Privacy. p. 113124. CPS-SPC 16, Association for Computing Machinery, New York, NY, USA (2016). https://doi.org/10.1145/2994487.2994494, https://doi.org/10.1145/2994487.2994494

10. iTrust: iTrust Datasets, https://itrust.sutd.edu.sg/itrust-labs_datasets/ 
11. Kandasamy, N.K.: An investigation on feasibility and security for cyber attacks on generator synchronization process. IEEE Transactions on Industrial Informatics (2019)

12. Mathur, A.P., Tippenhauer, N.O.: Swat: a water treatment testbed for research and training on ics security. In: CySWater. pp. 31-36 (April 2016)

13. Perales Gmez, .L., Fernndez Maim, L., Huertas Celdrn, A., Garca Clemente, F.J., Cadenas Sarmiento, C., Del Canto Masa, C.J., Mndez Nistal, R.: On the generation of anomaly detection datasets in industrial control systems. IEEE Access 7, 177460-177473 (2019)

14. Rajkumar, R., Lee, I., Sha, L., Stankovic, J.: Cyber-physical systems: The next computing revolution. In: Design Automation Conference. pp. 731-736 (6 2010)

15. Siddiqi, A., Tippenhauer, N.O., Mashima, D., Chen, B.: On practical threat scenario testing in an electric power ics testbed. In: Proceedings of the 4th ACM Workshop on Cyber-Physical System Security. pp. 15-21 (2018)

16. Sugumar, G., Mathur, A.: A method for testing distributed anomaly detectors. International Journal of Critical Infrastructure Protection 27, $100324 \quad$ (2019). https://doi.org/https://doi.org/10.1016/j.ijcip.2019.100324, http://www.sciencedirect.com/science/article/pii/S1874548219301210

17. Sutton, F.: An Efficient Platform and Communication Architecture for Event-triggered Cyber-physical Systems. Ph.D. thesis, ETH Zurich (2018). https://doi.org/10.3929/ethz-b000260384

18. US-CERT: Crashoverride malware. US-CERT Report (2017), https://www.uscert.gov/ncas/alerts/TA17-163A

\section{A Supporting Data}




\begin{tabular}{|c|c|}
\hline IP Address & Device \\
\hline 172.16 .1 .41 & Generation stage PLC \\
\hline 172.16.2.41 & Transmission stage PLC \\
\hline 172.16 .3 .41 & Microgrid stage PLC \\
\hline 172.16.4.41 & Smart Home stage PLC \\
\hline 172.16 .5 .41 & Control stage PLC \\
\hline 172.18 .5 .60 & SACDA System \\
\hline 172.18 .5 .100 & Historian Server \\
\hline 172.16 .2 .11 & Transmission $T I E D_{1}$ \\
\hline 172.16 .2 .12 & Transmission $T I E D_{2}$ \\
\hline 172.16 .2 .13 & Transmission $T I E D_{4}$ \\
\hline 172.16 .3 .11 & Microgrid $M I E D_{1}$ \\
\hline 172.16 .3 .12 & Microgrid $M I E D_{2}$ \\
\hline 172.16 .4 .11 & Smart Home $S I E D_{1}$ \\
\hline 172.16 .4 .12 & Smart Home $S I E D_{2}$ \\
\hline 172.16 .4 .13 & Smart Home $S I E D_{3}$ \\
\hline 172.16 .4 .14 & Smart Home $S I E D_{4}$ \\
\hline 172.16 .1 .11 & Generation $G I E D_{1}$ \\
\hline 172.16 .1 .12 & Generation $G I E D_{2}$ \\
\hline
\end{tabular}

Table 2: IP addresses for key devices in the network. 